\title{
The Ligamentum Flavum at L4-5: Relationship With Anthropomorphic Factors and Clinical Findings in Older Persons With and Without Spinal Disorders
}

\author{
Andrew J. Haig, MD, Adodeji Adewole, MD, Karen S. J. Yamakawa, MS, \\ Benjamin Kelemen, BS, Andrea L. Aagesen, DO
}

Objective: To examine the relationship between ligamentum flavum thickness and clinical spinal stenosis.

Design: A validation study.

Setting: Clinical research laboratory.

Patients: A total of 119 subjects from the Michigan Spinal Stenosis Study (MSSS).

Methods: Two new measurement techniques were compared by use of magnetic resonance images of 4 asymptomatic subjects by 2 examiners. The technique with the best interrater reliability was then used to measure the ligamentum flavum at L4-L5 in 119 subjects in the MSSS who, on the basis of clinical examination without imaging, were thought to have lumbar stenosis, mechanical back pain, or no pain. These findings were related to other radiologic findings, demographics, clinical severity, and electrodiagnostic findings.

Main Outcome Measurements: Perpendicular on the inside of the spinal canal from the deepest point of concavity of the lamina to the edge of the ligament.

Results: The ligamentum flavum width measurement had high interrater $(r=0.774)$ and intrarater $(r=0.768)$ reliability. In 28 asymptomatic volunteers, ligamentum flavum width averaged $5.72 \pm 0.95 \mathrm{~mm}$, with the left side significantly thinner than the right $(\mathrm{t}=2.117$, $P=.044)$, and thicker ligaments with age $(r=0.653, P<.001)$. Asymptomatic persons whom radiologists thought had stenosis had thicker ligaments $(t=2.273, P=.032)$. Persons with clinical stenosis $(n=48)$ and mechanical pain $(n=43)$ had ligament thickness similar to that of asymptomatic volunteers. Among patients with clinical stenosis, ligamentum flavum thickness did not relate to symptom severity (pedometer and laboratory ambulation tests, Pain Disability Index, and visual analog scale for pain). Most neurophysiological findings had no relationship with ligamentum flavum width, except the presence of limb fibrillation potentials related to a thinner ligament $(t=2.915, P=.004)$.

Conclusions: The measurement technique is standardized for the ligamentum flavum for future use. Although the ligamentum flavum appears to get thicker with age, other factors, including clinical diagnosis, pain, and function, do not appear to relate to the ligamentum flavum width.

PM R 2012;4:23-29

\section{INTRODUCTION}

The ligamentum flavum, which envelops the medial aspect of the facet joints, is thought to play a role in spinal disorders. This role was first hypothesized by Elsberg in 1913 when he noted that a torn ligamentum flavum can "compress the fourth lumbar root" [1]. Since then, others have suggested that the ligamentum flavum plays an important role in the spinal disease, most prominently spinal stenosis [2-5].

To date, most authors describe the ligamentum flavum in histological [6-8], biochemical $[4,7,9]$, or biomechanical $[6,7]$ terms. Accurate measurement of the ligamentum flavum can help us to understand its impact on spinal disorders. Anatomical measurements of ligamentous thickness are less commonly used, and the techniques used for measurement are rarely described or validated $[6-8,10]$. Experience in the assessment of other aspects of spinal

A.J.H. The University of Michigan Department of Physical Medicine and Rehabilitation, 325 E. Eisenhower, Ann Arbor, Ml 48108. Address correspondence to A.J.H.; e-mail: andyhaig@ umich.edu

Disclosure: 1B, president, Haig et al Consulting; $3 \mathrm{~A}$, various academic organizations; $5 \mathrm{~A}$; 7B, Center for Healthcare; 8B, NIH R-O1 for Spinal Stenosis

A.A. The University of Michigan Department of Physical Medicine and Rehabilitation, Ann Arbor, Ml

Disclosure: nothing to disclose

K.S.J.Y. The University of Michigan Department of Physical Medicine and Rehabilitation, Ann Arbor, Ml

Disclosure: nothing to disclose

B.K. The University of Michigan Department of Physical Medicine and Rehabilitation, Ann Arbor, MI

Disclosure: nothing to disclose

A.L.A. The University of Michigan Department of Physical Medicine and Rehabilitation, Ann Arbor, Ml

Disclosure: nothing to disclose

Dr. Adewole was supported by the Globus Fellowship of the International Society for the Study of the Lumbar Spine. The Michigan Spinal Stenosis Study was supported by Award Number R01HD059259 from the Eunice Kennedy Shriver National Institute of Child Health \& Human Development. The content is solely the responsibility of the authors and does not necessarily represent the official views of the Eunice Kennedy Shriver National Institute of Child Health \& Human Development or the National Institutes of Health.

Peer reviewers and all others who control content have no relevant financial relationships to disclose.

Submitted for publication January 13, 2011; accepted August 25, 2011 
anatomy has shown that it is important to make measurements in populations with and without clinical symptoms.

The authors of the Michigan Spinal Stenosis Study (MSSS) gathered magnetic resonance images along with clinical examinations and electrodiagnostic (EDX) parameters in a population of persons with clinically evident spinal stenosis, mechanical back pain, or no spine symptoms $[11,12]$. This population can be used to describe the normal ligamentum flavum and to explore the relationships between ligament thickness and clinical findings. In the current study, we design and validate a technique for measurement of the thickness of the ligamentum flavum. This measurement technique is applied to a previously gathered, well-documented population of older persons with no symptoms, low back pain, or clinically apparent spinal stenosis.

\section{METHODS}

In summary, 2 different techniques for measuring ligamentum flavum thickness were designed and tested for interrater and test-retest validity. The most reliable of these was used in the clinical study. In the clinical study, investigators evaluated ligamentum flavum thickness in 3 groups of persons who had no symptoms, signs and symptoms of mechanical back pain without stenosis, or symptoms of lumbar spinal stenosis. The relationship between ligamentum flavum thickness and clinically relevant parameters was explored. These parameters include anthropomorphic data; masked clinical, radiological, and EDX impressions; and functional status, including laboratory walking tests. The study was approved by the university's institutional review board.

\section{Part 1: Design of a Ligamentum Flavum Measurement Technique}

To choose the optimal technique of measuring the thickness of the ligamentum flavum, magnetic resonance imaging (MRI) of 4 asymptomatic subjects were arbitrarily selected from a larger study population (MSSS, described in Part 2, Methods section, below) by a person who had no knowledge of the MRI findings. Two different techniques were used to measure the ligamentum flavum on the right and left side at 5 levels (L1-L5).

In method A (Figure 1), a perpendicular line is drawn down the midline of the spinal canal for reference. Two additional lines are drawn, one from the reference line to the anterior tip of the facet joint that is closest to the spinal canal, at a right angle to the reference line, and the other from the edge of the spinal canal along the initial line to the same point in the reference line, also at a right angle. One measurement is subtracted from the other to determine a measurement of ligamentum flavum thickness.

In method B, also shown in Figure 1, the curvature of the internal border of the lamina is noted. At the deepest point of

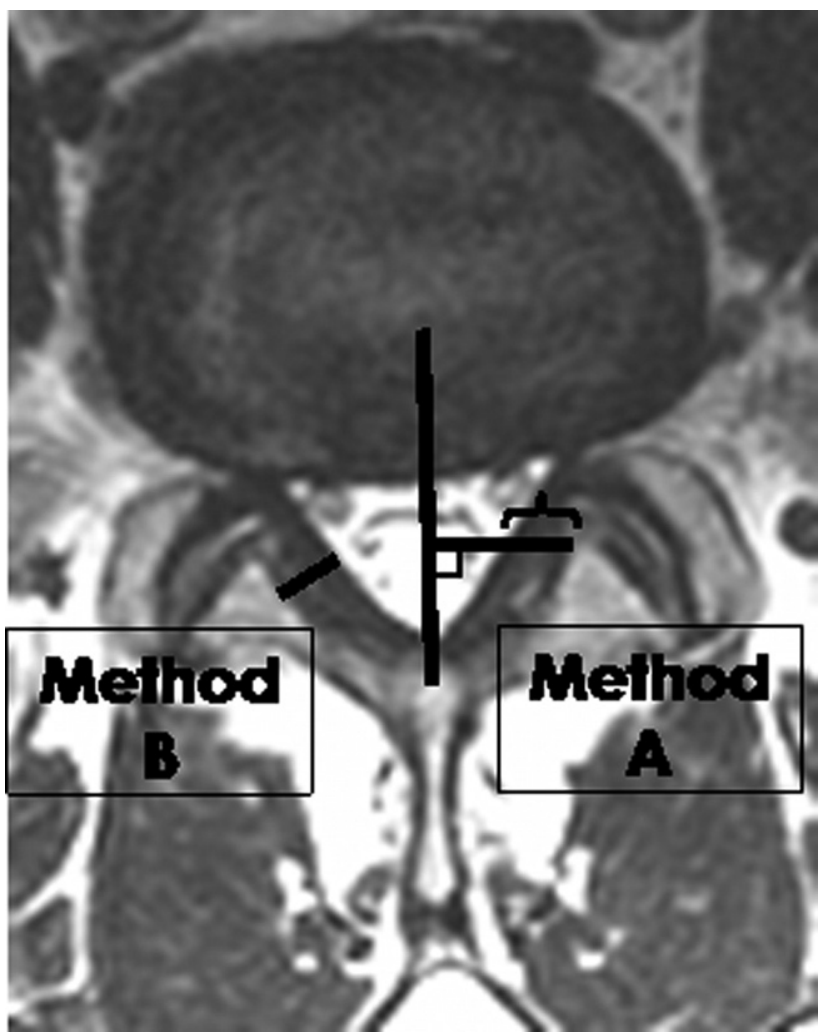

Figure 1. Ligamentum flavum measurement, methods $A$ and $\mathrm{B}$.

the curvature, a perpendicular is dropped to the surface of the ligamentum flavum. This perpendicular, usually the thickest part of the ligament, is the recorded measurement.

At times, it was difficult to precisely identify the anatomical landmarks required for measurement because of degenerative changes, shadows on the image, or the location of the slice. In these cases, 2 different techniques for finding the landmarks were used. The first was to look at the previous and subsequent slice for a clearer picture. In cases in which it was difficult to identify the deepest point of the curve in the lamina for method $\mathrm{B}$, the location of the deepest point of the curve was confirmed by looking at the spinal canal on the other side of the ligamentum flavum; to a smaller extent, the spinal canal mirrors the dip in the lamina with a smaller protrusion.

Measurements were taken on 5 levels, L1/L2 to L5/S1, by the use of axial T2-weighted lumbar spine MRI. The criteria for deciding which MRI slice at each level was measured were defined according to 3 variables: the view of the disk afforded by the slice, the formation of the inferior facet joint, and the formation of the lamina. Initially, the slice that cut most directly through the disk was used. However, in the case in which there were 2 slices with relatively similar views of the disk, the slice that fit 2 of the 3 criteria was used. The first 2 sets of intrarater reliability measurements were performed by an undergraduate student who had no previous radiological 
experience. The second set of interrater reliability measurements was performed by a fourth-year medical student with some previous radiological experience. Each set of measurements was performed 24 hours apart. The reliability of 2 different methods for measuring the ligamentum flavum was then tested.

\section{Part 2: Subjects, Clinical Measures, and Ligamentum Flavum Measures}

The data for the current study were obtained from the MSSS. Methods for this prospective diagnostic and prognostic trial are described in detail elsewhere [12]. In brief, after we screened for confounding disease, including polyneuropathy, 139 subjects underwent extensive history and physical examination by musculoskeletal physiatrists who were blinded to any MRI or electromyography results. On the basis of this clinical information, the physiatrists characterized the subjects as having no back pain, mechanical back pain only, or clinical spinal stenosis. EDX and MRI were performed by blinded clinicians. For the purpose of the current article, subjects who were subsequently found on EDX to have polyneuropathy or who had missing or invalid diagnostic tests were removed from the analysis, leaving 119 cases.

MRI studies on all subjects included a non-contrastenhanced lumbosacral spinal scan (GE Signa Horizon LX; GE Medical Systems, Milwaukee, WI), along with sagittal T2weighted (field of view [FOV]: 30; scan thickness [ST]: 3.0 mm, interscan spacing [IS]: $0.5 \mathrm{~mm}$, matrix: $384 \times 192$; repetition time [TR]: 3000; and echo time [TE]: 102 pulse: fast spin echo), sagittal T1-weighted (FOV: $30 ; \mathrm{ST}: 3 \mathrm{~mm}$, IS: $0.5 \mathrm{~mm}$, matrix: $256 \times 192$, TR: $400-700$, TE: min full pulse: spin echo), and axial T2-weighted (FOV: 20; ST: 4 mm; IS: 5 $\mathrm{mm} ; 5$ slices through each disk space T12-L1 through L5-S1; matrix: $256 \times 256$; TR: 3000-5000; TE: 102 pulse: FSE) scans. All images were reviewed at a workstation (Windows Advantage Workstation; GE Medical Systems). A neuroradiologist, who was blinded to clinical and electromyography (EMG) data, performed numerous measurements of the spinal segments and provided a clinical impression of stenosis or no stenosis.

EDX was performed by physiatrists who were board certified or eligible in EDX medicine and who were blinded to imaging and clinical information. Needle EMG was performed on 5 limb muscles on the clinically most painful side or a randomly chosen side if symmetrical or asymptomatic. Paraspinal mapping quantitative needle EMG of the L2-L5 innervated multifudus muscle was performed bilaterally. Sural sensory, peroneal motor, and H-wave nerve conduction studies were performed, all with the use of standard techniques. For the purposes of this study, the presence of $1+$ fibrillation potentials in any limb muscle and a paraspinal mapping score of greater than 4 were considered abnormal.
Demographic and clinical data were obtained from the patient questionnaire. In addition, ambulation velocity in a 15-minute walking test and the number of steps taken in 7 days as measured by a pedometer (DIGI-WALKER, Model SW-701; Yamax, Tokyo, Japan) were collected.

A physician who had not seen the images previously and who was masked to the clinical diagnosis performed all subsequent measures by using Imagecast iPACS Viewer, version 10.3.9.91 (IDX Systems Corporation, South Burlington, VT). He first selected the midline sagittal T2-weighted magnetic resonance images of the lumbar spine at the L4-L5 interspace. Method B, which was shown in the previously mentioned experiment to be more reproducible, was used for all measurements.

Data were initially entered into an Excel (Microsoft Corp., Redmond, WA) spreadsheet and checked for errors. SPSS version 15.0 (SPSS Inc., Chicago, IL) was used to analyze the results after importing the cleaned Excel data into SPSS. A one-sample Kolmogorov-Smirnov test was conducted to test the normality of the distribution of the measurements of the ligamentum flavum (right, left, and the average of right and left). An independent-samples $t$-test was used to determine mean difference between 2 groups, and one-way analysis of variance was used for comparing the mean differences among 3 groups. The chi-square test of independence was used to examine group differences for categorical measures. Tests of strength of association between ligamentum flavum with demographics, pain, ambulation, and MRI anatomical measures were assessed by the use of Pearson correlation analysis. An alpha value of 0.05 was used for all statistical analyses.

\section{RESULTS}

\section{Study Part 1: Design of a Ligamentum Flavum Measurement Tool}

All subjects tended to have similar mean widths across all 4 levels when method A was used, ranging from 6.09 to 8.03 $\mathrm{mm}$, whereas a larger range of measurements, from 3.31 to $6.08 \mathrm{~mm}$, was observed with method B. However, method A had a larger standard deviation than method B. Because the measurements were examined from level to level, the mean width tended to increase from L1/L2, with the largest mean at L4/L5 when both measurement types were used. The total mean values for all subjects at all levels tended to be larger for the right than for the left side when both measurement methods were used. The total means for all subjects across all levels and on both sides was slightly larger with method A than with method B, as was the standard deviation.

Method A was associated with poor intrarater reliability and statistically significant moderate interrater reliability, 0.58 , was statistically significant (Table 1 ). Method B was more reproducible, with intra- and interrater reliability statistically significant, both at 0.77 . 
Table 1. Measurement technique development: intra- and interrater reliability for ligamentum flavum measures on MRI with methods $A$ and method $B$ (4 subjects at 5 lumbar levels, right and left sides), $n=40$

\begin{tabular}{lcccc}
\hline & Width, mm & SD & ICC & P Value \\
\hline $\begin{array}{l}\text { Intrarater (rater 1), } \\
\quad \text { method A }\end{array}$ & & & 0.306 & .129 \\
$\quad \begin{array}{llll}\text { First measurement } \\
\quad \text { Second measurement }\end{array}$ & 6.875 & 2.062 & & \\
Interrater, method A & & 2.183 & & \\
$\quad$ Rater 1 & 6.875 & 2.062 & & \\
$\quad$ Rater 2 & 6.750 & 1.986 & & \\
$\begin{array}{l}\text { Intrarater (rater 1), } \\
\quad \text { method B }\end{array}$ & & & 0.768 & $<.001$ \\
$\quad$ Measurement 1 & 5.220 & 1.723 & & \\
$\quad$ Measurement 2 & 5.683 & 1.685 & & \\
Interrater, method B & & & 0.774 & $<.001$ \\
$\quad$ Rater 1 & 5.220 & 1.723 & & \\
$\quad$ Rater 2 & 4.643 & 1.446 & & \\
\hline
\end{tabular}

ICC, inter- or intraclass correlation coefficient.

Table 2 shows data measurements at a segmental level. Intrarater reliability reached significance $(P<.05)$ in only 2 of 10 measurements with method A, interrater reliability was significant in 6 of 10 measurements with method B. Regarding interrater reliability, no segmental measurement was statistically significant for method A, whereas 5 of 10 measurements were significant for method B.

\section{Study Part 2: Anthropomorphic and Clinical Relations to the Ligamentum Flavum}

The population used for the second part of the study had a mean age of $65.1 \pm 7.59$ years, and $32.8 \%(n=39)$ were male. The physiatrist's history and physical examination classified the subjects into 3 groups: asymptomatic $(n=28)$, mechanical back pain $(\mathrm{n}=43)$, and clinical spinal stenosis $(\mathrm{n}=48)$. The blinded radiologist believed that 69 subjects had radiological stenosis. These groupings were used for examining the relationships between the ligamentum flavum and various demographic, pain, and functional measures, and anatomical parameters at the corresponding level.

In the 28 asymptomatic subjects, the mean (left and right) ligamentum flavum thickness ( \pm standard deviation) was $5.72 \pm 0.95 \mathrm{~mm}$. The right $(5.89 \pm 1.12 \mathrm{~mm})$ was significantly thicker than the left side $(5.55 \pm 0.94 \mathrm{~mm}$; mean difference of $0.34 \pm 0.84 \mathrm{~mm}$, paired $t$-test, $\mathrm{t}=2.117, P=$ .044). Table 3 describes the relationship between ligamentum flavum thickness and various demographic, clinical, and radiological measures. The ligamentum flavum width was positively correlated with age $(r=0.653, P<.001)$ but was inversely related to the anteroposterior thecal sac diameter $(\mathrm{r}=-0.444, P=.018)$ and thecal sac area $(\mathrm{r}=-0.433, P=$ $.021)$. The ligamentum flavum was significantly thicker in the 15 asymptomatic volunteers whom radiologists thought had stenosis compared with the 13 whom they believed did not have stenosis $(6.07 \pm 1.03 \mathrm{~mm}$ vs $5.31 \pm 0.67 \mathrm{~mm}, \mathrm{t}=$ $2.273, P=.032$ ). All other measures showed no significant relationship to ligamentum flavum width.

Table 4 seeks relationships between the 3 clinically defined populations and ligamentum flavum thickness. Of 69 persons whom the radiologist believed had stenosis, 29 had clinically defined stenosis, 25 had low back pain, and 15 were asymptomatic. No relationship was observed between ligament thickness and the clinical diagnosis of stenosis.

Among persons who did appear to have clinical spinal stenosis, no relationship was found between the L4-L5 ligamentum flavum width (averaged left and right) and any of the measures of clinical severity (pedometer walking distances per day or per week, 15-minute ambulation velocity, Pain

Table 2. Measurement technique development: Intra- and interrater reliability for ligamentum flavum measures at each lumbar level with techniques $A$ and $B$ in the MRI scans of 4 subjects

\begin{tabular}{|c|c|c|c|c|c|c|c|c|c|c|}
\hline & \multicolumn{2}{|c|}{$\mathbf{L 1} / \mathbf{L} 2$} & \multicolumn{2}{|c|}{$\mathrm{L} 2 / \mathrm{L3}$} & \multicolumn{2}{|c|}{ L3/L4 } & \multicolumn{2}{|c|}{ L4/L5 } & \multicolumn{2}{|c|}{ L5/S1 } \\
\hline \multicolumn{11}{|l|}{$\begin{array}{l}\text { Method A measurement } \\
\text { Right side }\end{array}$} \\
\hline Intrarater (rater 1) & 0.935 & .025 & 0.672 & .192 & 0.827 & .588 & 0.523 & .279 & -2.725 & .846 \\
\hline Interrater (raters 1 and 2) & 0.825 & .093 & 0.289 & .393 & 0.092 & .243 & 0.606 & .232 & -2.362 & .827 \\
\hline \multicolumn{11}{|l|}{ Left side } \\
\hline \multicolumn{11}{|l|}{$\begin{array}{l}\text { Method B measurement } \\
\text { Right side }\end{array}$} \\
\hline Intrarater (rater 1) & 0.963 & .012 & 0.474 & .306 & 0.994 & .001 & 0.903 & .043 & 0.283 & .396 \\
\hline Interrater (raters 1 and 2) & 0.836 & .086 & 0.650 & .206 & 0.931 & .027 & 0.969 & .009 & -0.317 & .587 \\
\hline \multicolumn{11}{|l|}{ Left side } \\
\hline Intrarater (rater 1) & 0.959 & .013 & 0.261 & .405 & 0.981 & .004 & 0.893 & .050 & 0.461 & .312 \\
\hline
\end{tabular}

ICC = inter- or intraclass correlation coefficient; MRI = magnetic resonance imaging 
Table 3. Relationships between ligamentum flavum thickness and various factors in 28 asymptomatic volunteers

\begin{tabular}{|c|c|c|c|}
\hline & Population, Mean (SD) & $\begin{array}{c}\text { Test Statistics R (Unless } \\
\text { Noted Otherwise) }\end{array}$ & $\begin{array}{l}\text { Significance } \\
\text { (P Value) }\end{array}$ \\
\hline \multicolumn{4}{|l|}{ Demographic measures } \\
\hline Height, cm & $168.00(10.08)$ & -0.095 & .630 \\
\hline Body mass index & $28.53(5.93)$ & 0.237 & .225 \\
\hline Age, years & $64.82(8.02)$ & 0.653 & $<.001$ \\
\hline \multicolumn{4}{|l|}{ Gender } \\
\hline Education, years & $15.95(3.24)$ & -0.027 & .912 \\
\hline \multicolumn{4}{|l|}{ Clinical factors } \\
\hline \multicolumn{4}{|l|}{ Radiologist diagnosis of stenosis } \\
\hline Stenosis & $6.07(1.03) n=15(53.6 \%)$ & $\mathrm{T}=2.273$ & .032 \\
\hline No stenosis & $5.31(0.67) n=13(46.4 \%)$ & & \\
\hline Paraspinal mapping EMG score & $1.39(2.33)$ & 0.202 & .304 \\
\hline \multicolumn{4}{|l|}{ Strength deficit } \\
\hline Deficit & $7.48(0.67) n=2(7.1 \%)$ & * & \\
\hline No deficit & $5.58(0.83) n=26(92.9 \%)$ & & \\
\hline \multicolumn{4}{|l|}{ Reflex deficit } \\
\hline Deficit & $5.39(1.22) n=7(25.0 \%)$ & $\mathrm{T}=1.052$ & .303 \\
\hline No deficit & $5.83(0.84) n=21(75.0 \%)$ & & \\
\hline Velocity $(\mathrm{km} / \mathrm{h})$ 15-minute walk test & $4.41(0.87)$ & -0.264 & .175 \\
\hline Steps per day (pedometer) & $6324.12(3453.93)$ & -0.307 & .112 \\
\hline Pain (PDI) & $0.85(1.90)$ & 0.127 & .528 \\
\hline Pain (VAS) & $0.19(0.51)$ & -0.013 & .951 \\
\hline \multicolumn{4}{|l|}{ Anatomical factors } \\
\hline \multicolumn{4}{|l|}{ Disk herniation at L4-L5? } \\
\hline Yes & $5.50(-) n=1(3.6 \%)$ & * & \\
\hline No & $5.73(0.96) n=27(96.4 \%)$ & & \\
\hline
\end{tabular}

$\mathrm{AP}=$ anteroposterior; $\mathrm{EMG}$ = electromyography; PDI = Pain Disability Index; VAS = visual analog scale.

*Statistical testing was not performed because of low count in one or more group(s).

Disability Index score, and visual analog scale score for pain), with $P>.15$ in all instances.

EDX evidence for nerve involvement was compared with ligamentum flavum width on the same side as the limb that underwent EDX testing. Interestingly fibrillations in the limb muscles related to a somewhat smaller ligamentum flavum: of 119 EMG studies, 24 had fibrillation potentials, and their ligaments averaged $4.88 \mathrm{~mm}( \pm 0.78)$, whereas the remaining 95 subjects had a ligament width of $5.69 \mathrm{~mm}( \pm 1.31)$, $\mathrm{t}=2.915, P=.004$. All other measures, including motor

Table 4. Relationship between ligamentum flavum width and clinical diagnosis

\begin{tabular}{|c|c|c|c|c|c|c|}
\hline $\begin{array}{l}\text { Ligamentum } \\
\text { Flavum }\end{array}$ & $\begin{array}{l}\text { All Subjects, Mean } \\
\text { (SD), } n=119\end{array}$ & $\begin{array}{l}\text { Clinical Stenosis, } \\
n=48\end{array}$ & $\begin{array}{l}\text { Mechanical Back Pain, } \\
\qquad n=43\end{array}$ & $\begin{array}{l}\text { Asymptomatic, } \\
n=28\end{array}$ & $\begin{array}{l}\text { Student T-test / } \\
\text { F-statistic }\end{array}$ & $P$ Value \\
\hline Right & $5.61(1.16)$ & $5.47(1.16)$ & $5.59(1.17)$ & $5.89(1.12)$ & 1.153 & .319 \\
\hline Left & $5.53(1.32)$ & $5.52(1.56)$ & $5.53(1.27)$ & $5.55(0.94)$ & 0.004 & .996 \\
\hline $\begin{array}{l}\text { Average right } \\
\text { and left }\end{array}$ & $5.57(1.13)$ & $5.50(1.23)$ & $5.56(1.16)$ & $5.72(0.95)$ & 0.338 & .714 \\
\hline
\end{tabular}


unit changes in the leg, paraspinal mapping, and H-wave testing, showed no relationship to ligament width.

\section{DISCUSSION}

The width of the ligamentum flavum is commonly noted on imaging studies; however, until this study, there has not been a validated quantitative measure, and normative values have not been published for MRI findings in older persons. This study provides these data and seeks association between ligamentum flavum thickness and clinically relevant factors.

\section{Design of a Ligamentum Flavum Measurement Tool}

In this part of the study, we found that method A was mathematically precise but tangential to the ligament thickness, resulting in potential errors. Method B appeared to require some judgment, but the judgment may not be critical, as reliability was good even when measured by an undergraduate and a medical student. Method B resembled the method used by Fukuyama et al [6]. However, the location measured by Fukuyama et al [6] was based on a determination of the thickest part of the ligament, which can be subjective. We used bony landmarks to decrease that subjectivity.

For method B, we found both good interrater measurements and good intrarater measurements. However, for method A, we found moderate interrater and poor intrarater measurements. Method B has additional strength because of the extent to which it reflects only the width of the ligament. Although method A reflects the width of the ligament, it reflects a component of the ligament length as well. Method A also requires twice as many measures as method B, which introduces twice the potential for measurement errors. We advocate use of method B in future studies of the ligament thickness.

\section{Anthropomorphic Measures}

Evaluation of the ligamentum flavum in asymptomatic volunteers can provide normative data and help us to understand factors that may cause the ligament to thicken. The population represented a range of heights and body mass indexes, which resulted in a reasonable range of measurement possibilities. Because the ligamentum flavum thickness increases with age [13] (and the spinal canal does not compensate by getting larger), it makes sense that the thecal sac measurements would decrease as the ligament increases. In the current study, we could not determine whether this increase in thickness with age relates to hypertrophy (perhaps in response to stressors) or to redundancy (perhaps related to relaxation of the ligament as the intervertebral space becomes smaller with disk collapse). The significantly thicker right-sided ligament may relate somehow to the fact that most persons are right handed; however, the mechanism for this relationship is not clear.

\section{Clinical Relationships With the Ligamentum Flavum}

Because the ligamentum flavum occupies the spinal canal, it is legitimate to question whether increased thickness of the ligamentum flavum has a relationship with the clinical syndrome of spinal stenosis. This relationship could develop in causal or coincident ways. As a direct cause, hypertrophy or redundancy could simply occupy space that the other canal contents need, causing stenosis symptoms. However, the hypertrophy or redundancy could be a marker for segmental hypermobility, and the hypermobility alone could then be the cause of stenosis. In this second instance, we would hope to see cases of stenosis symptoms in persons whose canals are "adequate" while they are lying supine in the scanner but whose ligaments are thicker than normal.

Another group recently examined ligamentum flavum thickness in an uncontrolled study of more than 200 MRIs; they concluded that redundancy is the reason for thickness [13]. Our data, however, demonstrate no good relationship between ligamentum flavum thickness and clinical diagnosis, level of pain, or level of disability. This finding parallels previously published analyses of numerous measures of the spinal canal and thecal sac diameter and area from the MSSS study [12]. In that report, a statistically significant relationship between the anteroposterior spinal canal diameter and clinically diagnosed stenosis was found; however, it had no discriminant value. The many other measures of the area and diameter of the canal and thecal sac did not relate to clinically diagnosed stenosis.

The radiologist reported spinal stenosis in a number of our clinically asymptomatic subjects. The current data indicate that this reporting behavior related to the thickness of the ligamentum flavum. This relationship may be an epiphenomenon related to a smaller thecal sac or the radiologists may consciously or subconsciously believe that a thicker ligament relates to stenosis. Regardless, the results of this study will encourage radiologists to avoid any conscious or subconscious inclusion of the ligamentum flavum thickness in drawing a conclusion about radiological stenosis.

It is curious that a thin ligamentum flavum correlated strongly with the presence of fibrillation potentials on the limb EMG examination. In the MSSS and a subsequent study, fibrillations on EMG had a very good relationship with the clinical presentation of stenosis and with a very low falsepositive rate $[12,14]$. Innervation has no known hypertrophic affect on ligaments. Perhaps, contrary to the previous discussion, a thin ligamentum flavum is actually the cause of, or a marker for, hypermobility.

The study methodology has a number of strengths in that it involves masked, validated measures in persons with a 
range of clinical presentations. However, for anthropometric purposes, it is important to note that 28 asymptomatic volunteers is a relatively small sample. A larger study may find more subtle positive relationships between the ligamentum flavum and factors such as obesity, body size, and even clinical presentation. We also only measured the ligamentum flavum at L4-L5 for the current study. However this is the most common level for radiological spinal stenosis to occur $[12,13]$

\section{CONCLUSION}

The ligamentum flavum can be measured accurately; however, within the limits of this study, measures of ligament thickness do not appear to relate to most clinically relevant information.

\section{REFERENCES}

1. Elsberg CA. Experiences in spinal surgery. Surg Gynecol Obstet 1913; 16:117-132.

2. Grenier N, Kressel HY, Schiebler ML, et al. Normal and degenerative posterior spinal structures: MR imaging. Radiology 1987;165:517-525.

3. Okuda T, Baba I, Fujimoto Y, et al. Pathology of ligamentum flavum in degenerative lumbar disease. Spine 2004;29:1689-1697.

4. Park JB, Chang H, Lee JK. Quantitative analysis of transforming growth factor-beta 1 in ligamentum flavum of lumbar stenosis and disc herniations. Spine 2001;26:492-495.

5. Yoshida M, Shima K, Taniguchi Y, Tamaki T, Tanaka T. Hypertrophied ligamentum flavum in lumbar spinal canal stenosis: Pathogenesis and morphologic and immunohistochemical observation. Spine 1992;17: 1353-1360.
6. Fukuyama S, Nakamura T, Ikeda T, Takagi K. The effect of mechanical stress on hypertrophy of the lumbar ligamentum flavum. J Spinal Disord Techniques 1995;8:126-130.

7. Sairyo K, Biyani A, Goel V, et al. Pathomechanism of ligamentum flavum hypertrophy: A multidisciplinary investigation based on clinical, biomechanical, histologic, and biologic assessments. Spine 2005; 30:2649-2656.

8. Schrader PK, Grob D, Rahn BA, Cordey J, Dvorak J. Histology of the ligamentum flavum in patients with degenerative lumbar spinal stenosis. Eur Spine J 1999;8:323-328.

9. Park JB, Lee JK, Park SJ, Riew KD. Hypertrophy of ligamentum flavum in lumbar spinal stenosis associated with increased proteinase inhibitor concentration. J Bone Joint Surg Am 2005;87:2750-2757.

10. Schulte TL, Heidenreich JO, Schilling AM, et al. Comparison of metric analysis of spinal structures, exemplarily of the ligamentum flavum obtained with CT and MRI. Eur J Radiol 2004;52:224-228.

11. Haig AJ, Tong HC, Yamakawa KSJ, et al. Spinal stenosis, back pain, or no symptoms at all? A masked study comparing radiologic and electrodiagnostic diagnoses to the clinical impression. Arch Phys Med Rehabil 2006;87:897-903.

12. Haig AJ, Geisser ME, Tong HC, et al. Electromyographic and magnetic resonance imaging to predict lumbar stenosis, low-back pain, and no back symptoms. J Bone Joint Surg Am 2007;89:358-366.

13. Altinkaya N, Yildirim $T$, Demir $S$, Alkan $O$, Sarica F. Factors associated with the thickness of the ligamentum flavum; is ligamentum flavum thickening due to hypertrophy or buckling? Spine 2011;36:E1093E1097.

14. Yagci I, Gunduz OH, Ekinci G, Diracoglu D, Us O, Akyuz G. The utility of lumbar paraspinal mapping in the diagnosis of lumbar spinal stenosis. Am J Phys Med Rehabil 2009;88:843-851.

This CME activity is designated for 1.0 AMA PRA Category 1 Credit ${ }^{\mathrm{TM}}$ and can be completed online at me.aapmr.org. Log on to www.me.aapmr.org, go to Lifelong Learning (CME) and select Journal-based CME from the drop down menu. This activity is FREE to AAPMER members and $\$ 25$ for non-members.

\section{CME Question}

In the study by Haig et al, thickness of the ligamentum flavum measured with magnetic resonance imaging was significantly associated with:
a. walking velocity
b. increasing age
c. pain
d. ambulation 